\title{
Serotipos y resistencia antibiótica en Shigella spp aisladas de infecciones intestinales, Lima, 2012
}

\section{Serotypes and antibiotic resistance in Shigella spp. isolated from intestinal infections, Lima, 201}

\author{
César E. Guerrero Barrantes ${ }^{1}$, Alfredo Guillén O. ${ }^{1}$, Roberto Rojas L ${ }^{1}$, Nora Bravo ${ }^{2}$ \& Paola Muñoz ${ }^{1}$ \\ ${ }^{1}$ Universidad Nacional Federico Villarreal, Facultad de Tecnología Médica, Lima 10 \\ 2 Universidad Nacional Federico Villarreal, Facultad de Ciencias Naturales, Lima 10
}

DOI: https://doi.org/10.33017/RevECIPeru2013.0005/

\section{Resumen}

Se ha descrito que la distribución mundial de los serogrupos de Shigella no es igual en las distintas regiones. El objetivo es determinar los serotipos, la frecuencia de éstos y el patrón de resistencia a los antimicrobianos de los cultivos de Shigella spp. aislados de infecciones intestinales. Se evaluaron 75 cultivos de Shigella spp., identificados bioquímicamente y serológicamente, tanto su serogrupo como su serotipo, por aglutinación en lámina. Los patrones de resistencia antibiótica se determinaron mediante el método de difusión de disco en agar. De los 75 cultivos de Shigella, 54 fueron Shigella flexneri $(72 \%)$ y 21 Shigella sonnei $(28 \%)$. De los 54 cultivos de Shigella flexneri, el $48,15 \%$ resultó ser del serotipo $2 a$, seguidos por los serotipos 1 b y 6 con el $12,96 \%$ cada uno, luego el serotipo $3 a$ con $11,11 \%$ y por último los serotipos $1 \mathrm{a}$, $4 \mathrm{~b}$ y $2 \mathrm{~b}$, con $5,56 \%, 5,56 \%$ y $3,70 \%$, respectivamente. La resistencia antibiótica observada en los cultivos de Shigella, independientemente del serogrupo, fue muy frecuente para Sulfametoxazol Trimetoprim, ampicilina, cloranfenicol y tetraciclina; además, algunos cultivos fueron resistentes a Aztreonam, Furazolidona y Amoxicilina-Acido Clavulánico. Los serotipos de Shigella flexneri desde infecciones intestinales, en Lima, son $2 a-1 b-6-3 a-1 a-4 b-2 b$; el más frecuente es el $2 a$, seguido por el $1 b$ y 6 y el patrón de resistencia observado en Shigella spp, fue elevado para sulfametoxazol-Trimetoprim, Tetraciclina, Cloranfenicol y Ampicilina.

Descriptores: Shigella, serotypes, resistance.

\section{Abstract}

The global distribution of serogroups in Shigella is not equal across regions. The objective is to determine serotypes, the frequency and pattern of resistance to antimicrobial agents of cultures of Shigella spp. isolated from intestinal infections. The 75 cultures of Shigella spp., identified biochemically and serologically, were evaluated for serogroup and serotype, by agglutination on slide. Antibiotic resistance patterns were determined by disk agar diffusion method. Of the 75 strains of Shigella, 54 were Shigella flexneri (72\%) and 21 Shigella sonnei (28\%). Of the 54 strains of Shigella flexneri, $48,15 \%$ proved serotype $2 a$, while $12,96 \%$ corresponded to the $1 \mathrm{~b}$ and 6 serotypes one each, than the serotype $3 \mathrm{a}$ with $11,11 \%$, and finally the serotypes $1 \mathrm{a}, 4 \mathrm{~b}$ and $2 \mathrm{~b}$, with $5.56 \%, 5,56$ and 3,70\%, respectively. Antibiotic resistance observed in cultures of Shigella, regardless of the serogroup, was very frequent for Sulfametoxazol Trimetoprim, ampicillin, chloramphenicol, and tetracycline; in addition, some strains were resistant for Aztreonam, furazolidone and amoxicillin-Clavulanic acid. The serotypes of Shigella flexneri from intestinal infections, in Lima, are $2 a-1 b-$ 6- $3 a-1 a-4 b-2 b$; the most frequent is the $2 a$, followed by $1 b$ and 6 serotypes, and the resistance pattern observed in Shigella spp., was elevated to trimethoprim-sulfamethoxazole, tetracycline, chloramphenicol and ampicillin. 
Keywords: Shigella, serotypes, resistance.

\section{Introducción}

Shigella es un bacilo gram negativo perteneciente a la familia Enterobacteriaceae, se caracteriza por no fermentar la lactosa, ser inmóvil, no produce lisina descarboxilasa y raramente produce gas a partir de hidratos de carbono. Su identificación se basa en características bioquímicas y antigénicas, describiéndose cuatro especies, todas ellas pueden causar disentería, aunque con diferente gravedad.

Las distintas especies de Shigella constituyen la principal causa de disentería bacteriana, diarrea caracterizada por eliminación frecuente de heces conteniendo pus, sangre y/o moco (Anand, et.al., 1986). El ser humano es el único reservorio conocido de este agente. La mayoría de los casos ocurren en niños, en general transmitidos por contacto directo. Los brotes a gran escala, vinculados a alimentos, son más raros. A pesar de ello, constituye un importante problema de salud pública mundial, debido fundamentalmente a su elevada transmisibilidad, la emergencia de cepas resistentes a antimicrobianos y la falta de vacunas efectivas.

En nuestro medio y en los países en desarrollo, la especie más común causal de shigelosis, aislada de heces diarreica, es S. flexneri, mientras que en Estados Unidos las dos terceras partes de los casos de shigelosis son causados por Shigella sonnei, seguido por Shigella flexneri (CDC, 1999).

Estudios basados en el ADN indican que Escherichia y Shigella tienen un estrecho parentesco. Se sugiere que el gen que codifica el antígeno $\mathrm{O}$ de Shigella sonnei, anteriormente era cromosomal, el que ahora se encuentra en un plásmido, permitiéndose ventajas de adaptación ante el ambiente (Lai et.al.,1998). El género Shigella, así como la variante patógena E. coli Enteroinvasiva (ECEI), tienen fenotipos perdidos marcadamente similares, con reducción en el número de sustratos utilizados en relación a $\mathrm{E}$. coli comensal, siendo necesario un suplemento nutricional adicional (Lan et. al., 2004). La diferencia bioquímica entre ECEI y Shigella es la utilización del acetato y mucato como única fuente de carbono, donde el primero puede ser positivo para uno 0 ambos, mientras que Shigella generalmente es negativo.
Todas las especies presentan antígeno $\mathrm{O}$, termoestable y pueden o no poseer antígeno $\mathrm{K}$, termolábil. Este último no interviene en la serotipificación, pero puede interferir en la determinación del antígeno $\mathrm{O}$, lo cual se evita mediante la ebullición de la suspensión del cultivo.

Gracias al antígeno $O$ podemos clasificar a este género en cuatro serogrupos: el $\mathrm{A}$ como $\mathrm{S}$. dysenteriae, el B como S. flexneri, el C como S. boydii y el $\mathrm{D}$ como $\mathrm{S}$. sonnei.

Cada serogrupo puede subdividirse en tipos (serotipos), en base a variantes del antígeno $\mathrm{O}$, estos serotipos se designan mediante números arábigos. Se recomienda identificar los serogrupos y serotipos respectivos de acuerdo al esquema sugerido por la OMS (Terragno et. al., 2007; HPA, 2007).

La dosis infecciosa es baja: 10 a 100 organismos, la bacteria es transmitida por la ruta fecal-oral, a través de agua y alimentos contaminados, ésta sobrevive al ambiente gástrico (resistencia a la acidez estomacal) y se dirige al intestino grueso (Todar, 2009). La bacteria se adhiere y penetra la célula epitelial de la mucosa intestinal, se internaliza a través de un endosoma, se lisa ésta y se libera en el citoplasma, donde se multiplican intracelularmente y se diseminan a las células vecinas, trayendo como consecuencia degeneración del epitelio e inflamación de la lámina propia por muerte masiva de los macrófagos, descamación y ulceración de la mucosa, con sangrado y moco (Anand, et. al., 1986), dando la característica de la patología de shigelosis.

La shigelosis es una infección invasora cuya virulencia está codificada por plásmido de gran tamaño (120 MDa en S. sonnei y de 140 MDa en el resto de especies de Shigella) que contienen genes (operón Ipa) que codifican la producción de proteínas (antígenos de invasión plasmidial), como el IpaA, IpaB, IpaC e IpaD necesarias para la adhesión epitelial, invasión y expansión de la bacteria a los macrófagos de la mucosa. Las lesiones más pronunciadas son vistas en el área rectosigmoidal y la intensidad de la inflamación decrece en la dirección proximal (Mathan and Mathan , 1991). Dos formas de plásmido de virulencia (pINV) han sido demostrados en Shigella, el pINV A y el pINV B (Lan et. al , 2004).

Cuando la bacteria crece a $37^{\circ} \mathrm{C}$, las proteínas de virulencia (Vir) se activan, el Vir $\mathrm{F}$ induce la expresión de la proteína VirB y ésta a su vez activa los promotores de los genes ipa (antígeno de 
invasión plasmidial), mxi (proteínas de excreción de membrana) y spa (antígenos presentadores de superficie), permitiendo la expresión de los operones respectivos. Sus proteínas sintetizadas dan lugar al complejo Translocon Mxi-Spa, el que se activa permitiendo la secreción de las proteínas Ipa (IpaB, IpaC e IpaA), las cuales van a interactuar con la membrana de la célula hospedadora, desencadenado una cascada de señales celulares que permitiría la internalización de la bacteria a través de endosoma, así como también de su salida ya en el citoplasma (Todar, 2009).

La infección bacteriana y la lesión tisular, conlleva a la diarrea y patología típica de shigelosis, pero, particularmente las toxinas producidas por Shigella, como las enterotoxinas 1 y 2 , producen secreción de fluidos intestinal, además la toxina Shiga, producido sólo por S. dysenteriae serotipo 1 es citotóxica para una variedad de células y responsable del desarrollo de lesiones vasculares en el colon, riñones y sistema nervioso central (Mathan \& Mathan, 1991). Los síntomas consisten en dolor abdominal, vómitos, fiebre y diarrea, que puede variar desde una disentería (diarrea sanguinolenta, moco y pus) hasta una diarrea acuosa (en el caso de Sh. sonnei). La enfermedad dura entre 3 hasta 14 días.

Schroeder and Hilbi (2008), señala que las investigaciones de los últimos 25 años han revelado que un sistema de secreción tipo III (SST3) codificado por un gran plásmido es un factor de virulencia clave de Shigella flexneri. El SST3 determina las interacciones de S. flexneri con las células intestinales translocando consecutivamente dos set de proteínas efectoras en las células blanco. Así, Shigella controla la invasión intra e intercelular, la muerte de macrófagos, así como la respuesta inflamatoria del hospedador (Bernardini et. al., 1989). Un entendimiento de los mecanismos moleculares en la patogénesis de Shigella fomentaría el desarrollo de una vacuna eficiente y segura, que paralelamente con la mejora de la higiene frenaría las infecciones por este patógeno. Shigella causa anualmente en los países en desarrollo 163 millones de episodios de diarrea y un millón de muertes (Kotloff, et. al. 1999), donde la secuencia por frecuencia de las especies es S. flexneri, S. sonnei, S. boydii, y $S$. dysenteriae, señalando como serotipos frecuentes de $\mathrm{S}$. flexneri, al $2 \mathrm{a}$, seguido por 1b, 3a, 4a y 6 .

En un estudio de mortalidad por diarrea aguda en niños menores de 5 años realizado en la Ciudad Hospitalaria "Dr. Enrique Tejera" de la Ciudad de
Valencia, estado Carabobo, se aisló Shigella en el $19 \%$ de los niños fallecidos por diarrea, resultando además S. flexneri el serogrupo más frecuente (Pérez-Schael, et. al. 2007); posteriormente, en la misma localidad, Bellorin y col. (2008), en un programa de vigilancia de la diarrea aguda, encontraron un $27 \%$ para Shigella, distribuido como: S. flexneri $21 \%$, S. sonnei $5 \%$, S. boydii $0.6 \%$ y $\mathrm{S}$. dysenteriae $0,1 \%$; resaltándose que los serotipos más frecuente en el serogrupo Shigella flexneri fueron $2 \mathrm{a}(40 \%), 3 \mathrm{a}(24 \%) ; 2 \mathrm{~b}$ $(18 \%) ; 1$ a $(6 \%) ; 6(4 \%) ; 3 b(2 \%), 4 a(2 \%)$, variante "X" $(2 \%)$ y variante "Y" $(2 \%)$, y relaciona al serotipo 2 a con la severidad del episodio; demostrando también la alta resistencia antibiótica de S. flexneri frente a tetraciclina, ampicilina, cloranfenicol, amoxicilina y ácido clavulánico y sulfametoxazol trimetoprim. Aunque, en un brote ocurrido en una comunidad escolar en Colombia, Hidalgo y col. (2002), a través del Instituto Nacional de Salud de Bogotá, describen como agente causal a Shigella flexneri y como único serotipo implicado el 6.

En Argentina, Merino y col. (2004), estudiaron 132 cultivos de Shigella, de las cuales $29(22 \%)$ correspondieron a S. sonnei y 103 (78\%) correspondían a $\mathrm{S}$. flexneri, de estas últimas, 91 pertenecían al serotipo 2, ocho fueron del serotipo 6 , tres pertenecieron al serotipo 3 y una correspondió al serotipo 1. Sin embargo, Mota y col. (2005), en Uruguay, reportaron la prevalencia de Shigella flexneri, seguido de S. sonnei y S. dysenteriae, donde los serotipos frecuentes de la primera fueron $2 \mathrm{a}, 3 \mathrm{c}, 4,6$ y 1 .

En nuestro país, en el distrito La Victoria, Lima, Perales y col. (2002), reportan la frecuencia de Shigella como causal de diarrea aguda en niños menores de dos años, con un 4,8\%, después de Campylobacter, resaltando que $\mathrm{S}$. flexneri es la más frecuente seguida de S. sonnei; mientras que, un estudio epidemiológico realizado en la amazonía peruana por Kosek et. al. (2008), en heces de niños de la zona, reportaron que el $33.1 \%$ de los aislamientos de Shigella flexneri, fueron los serotipos $2 \mathrm{a}$, seguido por $3 \mathrm{a}$ con el $19.4 \%$, el 6 con $16.5 \%$, el serotipo 4 a con el $10.1 \%$ y los otros serotipos sumaron menos del 10\%. Asi mismo, reportan el nivel de resistencia antibiótica, donde se manifiesta la secuencia de frecuencia de resistencia con tetraciclina $(83 \%)$, sulfametoxazol trimetoprim (79\%), ampicilina (73\%) y cloranfenicol $(62 \%)$; sin embargo, los cultivos estudiados fueron sensibles a ceftriaxona, azitromicina, ácido nalidixico y ciprofloxacina. 
Se ha descrito que la distribución mundial de los serogrupos de Shigella no es igual en las distintas regiones; S. flexneri es más frecuente en los países en desarrollo, mientras que en los países desarrollados predomina S. sonnei (Kotloff et. al., 1999). Así como también la distribución de serotipos en el caso de S. flexneri, observándose algunas variantes en la frecuencia de éstos en Argentina (Merino y col., 2004), Colombia (Bellorin y col., 2008) y Uruguay (Mota y col., 2005). En nuestro medio y especialmente en Lima, no hay un conocimiento definido sobre la zonificación de los serotipos de Shigella que se encuentran causando infecciones intestinales y menos un mapeo regional o nacional. Pues, se ha descrito que la distribución mundial de los serogrupos de Shigella no es igual en las distintas regiones; así mismo la frecuencia de los serotipos de Shigella suelen mostrar variaciones. Es muy probable que en nuestro país se pueda dar diferencias en la distribución de los serotipos de Shigella, en las diferentes regiones, añadiéndose a esta problemática, el creciente desarrollo de multirresistencia a los antibióticos que presenta Shigella (Sack et. al., 1997; Hernández y col., 2002; CDC, 2006), lo que reduce considerablemente las opciones de tratamiento y enfoca la lucha contra la enfermedad hacia otro horizonte como es el desarrollo de vacunas (Coster et. al., 1999). Algunas de estas vacunas se orientan a estimular la respuesta inmune contra los determinantes antigénicos serotipos específicos del polisacárido "O" (Fries et. al., 2001) del lipopolisacárido (LPS) de la pared celular bacteriana. Pero el desarrollo de estos programas de vacunas solo tendrá éxito si se establecen en las regiones sistemas de vigilancia, que permitan conocer la prevalencia de la enfermedad y la distribución de los distintos serogrupos y serotipos de la bacteria. $E$ conocimiento detallado de la estructura antigénica resulta de utilidad para estudios epidemiológicos y en la formulación de vacunas.

Ante esto, surgen las preguntas ¿Cuáles son los serotipos de Shigella aislados desde infecciones intestinales, en Lima? y ¿cuáles son los más frecuentes?, así como ¿Cuál es el patrón de resistencia antibiótica presente en dichos cultivos? El objetivo del estudio es conocer los serotipos, la frecuencia de éstos y el patrón de resistencia a los antimicrobianos de cultivos de Shigella spp. aislados de infecciones intestinales, provenientes de tres centros de salud de Lima, 2012.

\section{Metodología}

\subsection{Método}

Es un estudio tipo prospectivo y descriptivo, basado en la detección de los serotipos y los patrones de resistencia antibiótica. Se evaluaron 75 cultivos de Shigella spp., aislados de infecciones intestinales, recolectados desde tres centros de salud de Lima, durante los meses de enero a noviembre, 2012, los cuáles fueron identificados bioquímicamente y serológicamente.

\subsection{Procedimiento}

\subsubsection{Toma de muestra}

Los cultivos de Shigella seleccionadas, aislados de infecciones intestinales en el Instituto de Salud del Niño, Hospital Madre - Niño San Bartolomé Herrera y Clínica San Borja, fueron inicialmente conservadas en Caldo Tripticasa de soya con Glicerol. Luego reactivadas por siembra en Agar Mac Conkey para determinar la pureza del cultivo y confirmadas como Shigella, usando el set de bioquímica de rutina para su identificación (HPA, 2007), siempre a partir de una colonia.

\subsubsection{Procesamiento de muestras}

\subsubsection{Determinación de los serogrupos y serotipos de Shigella (NPA, 2007; Terragno, 2007)}

Se sembraron desde una colonia, la cepa seleccionada, en Agar Tripticasa de Soya (TSA), tubo inclinado, e incubado por 24 horas a $37^{\circ} \mathrm{C}$.

El cultivo obtenido en el paso anterior fue resuspendido con $3 \mathrm{~mL}$ de Solución Salina Fisiológica estéril, obteniéndose una suspensión equivalente al 0,5 de la escala de Mc Farland.

Los sueros polivalentes de serogrupos y monovalentes respectivos alcanzaron la temperatura del ambiente.

Mezclamos cuidadosamente, sobre una lámina de vidrio, una asada de la suspensión bacteriana y una gota de solución salina fisiológica (control de aglutinación), se siguió con los pasos posteriores al no aglutinar.

Mezclamos cuidadosamente, sobre una lámina de vidrio, una asada de la suspensión bacteriana y una gota de antisuero polivalente, se movió la lámina suavemente por 2 minutos (la suspensión 
homogénea indicó la reacción negativa; mientras que la reacción positiva se evidenció por una aglutinación). Probamos en primera instancia, con el polivalente para S. flexneri; si no aglutinaba se probó con el suero para $S$. sonnei y así sucesivamente se seguió con los sueros para S. boydii y S. dysenteriae.

Los cultivos con característica bioquímica de Shigella, con aglutinación pobre 0 no, sus suspensiones fueron calentadas en baño maría a $100{ }^{\circ} \mathrm{C}$ por 30 minutos y se procedió al ensayo como se indica en la línea de arriba.

La aglutinación positiva de los \% cultivos con los antisueros polivalentes fueron evaluados posteriormente con sus respectivos monovalentes.

Se registraron los resultados obtenidos en la hoja respectiva.

\subsubsection{Resistencia Antibiótica - Resistencia Antibiótica}

Los patrones de resistencia antibiótica se determinaron mediante el método de difusión de disco en agar, siguiendo los lineamientos del Clinical and Laboratory Standards Institute (CLSI,2006). Se probaron los antibióticos: Ampicilina $(10 \mu \mathrm{g})$, amoxicilina / ácido clavulánico $(20 \mu \mathrm{g} / 10 \mu \mathrm{g})$, sulfametoxazol-trimetoprim $(1,25 \mu \mathrm{g} /$ $23,75 \mu \mathrm{g})$, tetraciclina $(30 \mu \mathrm{g})$, Cloranfenicol $(30 \mu \mathrm{g})$, ceftriaxona $(30 \mu \mathrm{g})$, cefotaxime $(30 \mu \mathrm{g})$, aztreonam $(30 \mu \mathrm{g})$, imipenem $(10 \mu \mathrm{g})$. Como control se usaron las cepas de E. coli ATCC 25922 y E. coli ATCC 35218.

Análisis Estadístico de los datos: Los datos obtenidos serán procesados siguiendo un patrón automatizado con el auxilio del software estadístico básico.

\section{Resultados y discusión}

De los setentaicinco (75) cultivos evaluados, que correspondieron bioquímicamente al género Shigella, cincuentaicuatro (54) fueron del grupo serológico B o Shigella flexneri, que representa el $72 \%(54 / 75)$ y veintiuno (21) fueron del grupo serológico D o Shigella sonnei, que corresponde al $28 \%(21 / 75)$, según podemos observar en la tabla № 1. Sólo se detectaron los dos serogrupos.
Tabla 1: Serogrupos Shigella

\begin{tabular}{|c|r|r|}
\hline SEROGRUPO & $\mathrm{n}$ & $\%$ \\
\hline Shigella B & 54 & 72 \\
Shigella D & 21 & 28 \\
\hline TOTAL & 75 & 100 \\
\hline
\end{tabular}

De los 54 cultivos de Shigella serogrupo B o Shigella flexneri, el 48,15\% (26/54) resultaron ser del serotipo $2 \mathrm{a}$, mientras que los serotipos $1 \mathrm{~b}$ y 6 fueron del 12,96 \% (7/54), cada uno, seguido el serotipo $3 a$ con $11,11 \%$ (6/54), luego por los serotipos 1 a y $4 b$, ambos con $5,56 \%(3 / 54)$ y por último el serotipo $2 b$ con el 3,7\% (2/54), como se demuestran en la tabla № 2 y el gráfico №1. Observándose que el serotipo con más frecuencia en este grupo de cepas estudiadas fue el serotipo 2a.

Tabla 2: Serotipificación Shigella B

\begin{tabular}{|l|r|r|}
\hline SEROTIPO & $\mathrm{n}$ & $\%$ \\
\hline B1a & 3 & 5,56 \\
\hline B1b & 7 & 12,96 \\
\hline B2a & 26 & 48,15 \\
\hline B2b & 2 & 3,70 \\
\hline B3a & 6 & 11,11 \\
\hline B3b & 0 & 0,0 \\
\hline B4a & 0 & 0,0 \\
\hline B4b & 3 & 5,56 \\
\hline B5a & 0 & 0,0 \\
\hline B5b & 0 & 0,0 \\
\hline B6 & 7 & 12,96 \\
\hline B Variante X & 0 & 0,0 \\
\hline B Variante $Y$ & 0 & 0,0 \\
\hline TOTAL & 54 & 100 \\
\hline
\end{tabular}

La resistencia antibiótica observada en los cultivos de Shigella, independientemente del serogrupo, fue muy frecuente para los antibióticos Sulfametoxazol Trimetoprim, ampicilina, cloranfenicol y tetraciclina, observándose dicho patrón en cultivos, tanto del serogrupo B (Shigella flexneri), como del serogrupo D (Shigella sonnei). Además, algunos cultivos expresaron resistencia a Aztreonam, Furazolidona y Amoxicilina-Acido Clavulánico, según se demuestran en la tabla № 3 .

Tabla 3: Resistencia antibiótica de shigella spp ( $n=75)$ 


\begin{tabular}{|c|c|c|c|c|c|c|c|c|c|c|}
\hline & Sxt & Te & Am & Cl & A-C & Fz & Az & Cef & Ctx & Im \\
\hline $\mathrm{n}$ & 75 & 71 & 48 & 53 & 4 & 4 & 4 & 0 & 0 & 00 \\
\hline$\%$ & 100 & 94 & 64 & 70 & 5 & 5 & 5 & 0,0 & 0,0 & 0,0 \\
\hline
\end{tabular}

Sxt: Sulfametoxazol trimetoprim; Te: Tetraciclina; Am: Ampicilina; Cl: Cloranfenicol; Fz: Furazolidona A-C: Amoxicilina-Ácido Clavulánico; Az: Aztreonam; Cef: Ceftriaxona; Ctx: Cefotaxima; Im: Imipinem; n: número de cultivos resistentes; \%: Porcentaje.

Como se puede observar en nuestros resultados, el serogrupo de Shigella más frecuente aislado es el B o Shigella flexneri (con el $72 \%$ ), seguido por Shigella sonnei o serogrupo D (con $28 \%$ ), como lo reporta Kotloff et. al (1999), en un estudio realizado sobre la carga mundial de infecciones por Shigella; así como también lo señalan Merino y col. (2004), en Argentina, quienes reportan que el $78 \%$ correspondió a Shigella flexneri; de igual manera Mota y col. (2005), en Uruguay y Bellorin y col. (2008), en Colombia, manifiestan el mismo sentido de la predominancia del serogrupo B sobre el D. Es importante señalar el estudio realizado por Kosek et. al. (2008), en la Amazonía peruana, quienes reportan el mismo sentido de frecuencia de serogrupo observado en nuestro estudio, donde el $67 \%$ fueron Shigella flexneri y el $11,8 \%$ fue Shigella sonnei, además de Shigella boydii $(11,4 \%)$ y Shigella dysenteriae $(2,4 \%)$, cuyas muestras fueron de heces provenientes de pacientes disentéricos o heces sanguinolentas, mientras que en nuestro estudio sólo se limitó a evaluar los cultivos de Shigella aislados de casos de infecciones intestinales y no necesariamente disentéricas.

De los 54 cultivos de Shigella flexneri (serogrupo B), evaluados para su serotipificación, se obtuvo la secuencia de frecuencia de serotipos: $2 a-1 b-6$ $-3 a-1 a-4 b-2 b$, donde el serotipo $2 a$ alcanzó el $48,15 \%$ (26/54), seguido por el 1 b y el 6 con $12,96 \%$ cada uno, mientras que el $3 a$ alcanzó el $11,11 \%$. Esta secuencia mantiene similitud con el serotipo $2 \mathrm{a}$, con el cual todos los autores coinciden en que es el de mayor presencia en este serogrupo; el resto de serotipos es de expresión variable en cuanto a su presencia y frecuencia, como podemos ver en la tabla № 4, donde Bollini y col. (2008), en Colombia, reportan los serotipos $2 b$ y 3b, mientras que Mota y col. (2005), en Uruguay, reportan el serotipo 3c, el cual no es descrito por los otros autores consultados y tampoco por nuestro estudio.

La secuencia de los serotipos de Shigella flexneri detectadas en el presente estudio es muy similar a la descrita por Kotlof (1999), con la frecuencia de los dos primeros serotipos; pero difiere un tanto a la secuencia reportada por Kosek et. al. (2008), a pesar de ser cultivos provenientes de nuestro país, pero de la región amazónica, resaltando la diferencia en el serotipo 1a (reportado con 5,56\%) y el $4 a$, donde el primero fue detectado sólo en nuestro estudio y el segundo fue detectado sólo en el trabajo de Kosek et. al (2008), además que el serotipo $1 \mathrm{~b}$, reportado con el $12,89 \%$ en el presente estudio, observa mayor frecuencia que el reportado $(9,7 \%)$ por dichos autores. Se deja entrever por los resultados alcanzados que el serotipo $2 \mathrm{a}$ es el de mayor frecuencia entre los cultivos de Shigella flexneri y los demás serotipos son variables.

Tabla 4: Comparación de serotipos de Shigella flexneri.

\begin{tabular}{|c|c|c|}
\hline AUTORES & $\begin{array}{c}\text { LUGAR/R } \\
\text { EGION }\end{array}$ & $\begin{array}{l}\text { SECUENCIA/ } \\
\text { SEROTIPOS }\left({ }^{*}\right)\end{array}$ \\
\hline Kotlof (1999) & Mundial & $2 a-1 b-3 a-4 a-6$ \\
\hline Merino (2004) & Argentina & $2-6-3-1$ \\
\hline Mota (2005) & Uruguay & $2 a-3 c-4-6-1$ \\
\hline Bellorin (2008) & Colombia & $\begin{array}{l}2 a-3 a-2 b-1 a-6-3 b- \\
4 a\end{array}$ \\
\hline Kosek (2008) & $\begin{array}{l}\text { Amaz./Per } \\
\text { ú }\end{array}$ & $2 a-3 a-6-4 a-1 b-4 b$ \\
\hline Guerrero (2012) & Lima/Perú & $\begin{array}{l}2 a-1 b-6-3 a-1 a-4 b- \\
2 b\end{array}$ \\
\hline
\end{tabular}

$\left.{ }^{*}\right)$ : La secuencia de serotipos indicada es por orden de mayor a menor frecuencia.

\section{Conclusiones}

1.- Los serotipos de Shigella flexneri detectados desde infecciones intestinales, en Lima son: $2 a-$ $1 b-6-3 a-1 a-4 b-2 b$.

2.- El serotipo más frecuente es el $2 \mathrm{a}$, seguido por los serotipos $1 \mathrm{~b}$ y el 6 .

3.- El patrón de resistencia observado en Shigella spp, fue elevado para SulfametoxazolTrimetoprim, Tetraciclina, Cloranfenicoly Ampicilina. 


\section{Agradecimientos}

Nuestro profundo agradecimiento a Yelina Vásquez y Doris Murga, alumnas de la Escuela del 5to. Año de Laboratorio de la Facultad de Tecnología Médica, por su valiosa colaboración. Así mismo, nuestro reconocimiento a los Srs. César Ventura y Herminio Pasache, personal técnico del Laboratorio por su gran apoyo.

\section{Referencias}

[1] B. S. Anand, V. Malhutra, S.K. Bhattacharya, P. Datta, D. Sen, M.K. Bhattacharya,S. Mukharjee and S. C. Pal. Rectal histology in acute bacillary Gastroenterology. 90 (1986) 654-660.

[2] I Bellorín; G. Urbina, F. González, B. Salinas, M. Tomat, R. González. Serotipos y Resistencia antimicrobiana de cepas de Shigella flexneri aisladas de niños con diarrea aguda. Relación entre el serotipo y la severidad del episodio. Rev. Soc. Venez. Microbiol. 28 (2008) 110-115

[3] M.L. Bernardini, J. Mounier, H D Hauteville, M. Coquis-Rondon. \& P. J. Sansonetti Identification of icsA, a plasmid locus of Shigella flexneri that governs intra and intercellular spread through interaction with factin. Proc Natl Acad. Sci USA, 86 (1989) 3867-3871.

[4] Center Diseases Control. Outbreaks of Shigella sonnei Infection Associated with Eating Fresh Parsley - United States and Canada, July-August 1998. MMWR. 48 (14) (1999)

[5] Center Diseases Control. Outbreaks of multidrug-resistant Shigella sonnei gastroenteritis associated with Day Care Centres Kansas, Kentucky, and Missouri, 2005. MMWR 55(39)1068-1071. (2006).

[6] Clinical and Laboratory Standards Institute/NCCLS. Performance standards for antimicrobial susceptibility testing. Sixteenth Informational Supplement. Clinical and Laboratory January Standards Institute, Wayne, Pa. USA. (2006).

[7] T.S Coster, C.W Hoge, L.L VanDeVerg, A.B Hartman, E.V Oaks, M.M Venkatesan, et. al. Vaccination against Shigellosis with attenuated Shigella flexneri 2a strain SC602. Infect Immun. 67(1999) 3437-43.

[8] LF Fries, AD Montemarano, CP Mallet, D.N Taylor, TL Hale, G.H Lowell. Safety and immunogenicity of a proteasome Shigella flexneri $2 a$ lipopolysaccharide vaccine administered intranasally to healthy adults. Infect. Immun. 69(2001): 4545-53.

[9] Health Protection Agency. Identification of Shigellas species National Standard Method BSOP ID. 20 Issue 2.(2007).

[10] M. Hidalgo, M. E. Realpe, N. Muñoz, D. Sicard, E. Silva, C.I. Agudelo, E. Castañeda. Brote de enfermedad diarreica aguda causado por Shigella flexneri en una escuela en Madrid, Cundinamarca: caracterización fenotípica y genotípica de los aislamientos. Biomédica (2002), 22 (003): $272-279$.

[11] M. Kosek, P. Peñataro, W. K. Pan, M. Paredes, R. H. Gilman, J. Perez, C. Banda, G. Meza, R. Burga and E- Hall. Epidemiology of Highly Endemic Multiply AntibioticResistant Shigellosis in Children in the Peruvian Amazon, Pediatrics;122(2008): e541-e549.

[12] K.L. Kotloff, J.P. Winickoff, B. Ivanoff, J.D. Clements, D.L, Swerdlow, P.J. Sansonetti, G.K. Adak and M.M. Levine. Carga mundial de infecciones por Shigella: implicaciones para el desarrollo de vacunas y la aplicación de estrategias de control. Bulletin WHO; 77 (1999) 651-66.

[13] V. Lai, L. Wang, and P. Reeves. Escherichia coli clone Sonnei (Shigella sonnei) had a chromosomal O-antigen gene cluster prior to gaining its current plasmid-borne O-antigen genes. J.Bacteriol.(1998). 180(11):29832986.

[14] R. Lan, M. Chehani, K. Donohoe, M.B. Martinez and P.R. Reeves. Molecular Evolutionary Relationships of enteroinvasive E. coli and Shigella spp. Infect. Immun. (2004). 72(9): 5080-5088.

[15] I. Hernández, \& G.A. Godoy. Shigella sp. Ais en Ciudad Bolivar. Prevalencia y su sensibilidad a los antimicrobianos. Rev. Soc Ven Microbiol. (2002). 22(1), pp. 22- 26.

[16] M.M. Mathan and V.I. Mathan. Morphology of rectal mucosa of patients with shigellosis. Rev.Infect.Dis. (1991). 13 [suppl. 4]: S314S318.

[17] M.I. Mota, G. Varela, M. Gadea, M.I. Caffer, A. Sirok \& F. Schelotto.

Serotipos, perfil plasmidico y antibiotipos de cepas de Shigella flexneri aisladas de niños menores de 5 años con diarrea sanguinolenta usuarios de los servicios de Salud Pública. Rev. Med. Urug. 21(2005):3036.

[18] L. A. Merino, G.E. Hreñuk, M.C Ronconi. y J.M. Alonso. Resistencia a antibióticos y epidemiología molecular de Shigella spp. en 
el nordeste argentino. Rev Panam Salud Publica. (2004). 15(4): 21924.

[19] M. Perales, M. Camiña, C. Quiñones. Infección por Campylobacter y Shigella como causa de Diarrea Aguda Infecciosa en niños menores de dos años en el Distrito de la Victoria, Lima-Perú. Rev. Per. med. exp. Salud pub.(2002) 19 (4) oct./dic.

[20] I. Pérez-Schael, B. Salinas, R González, H. Salas, J.E. Ludert, M. Escalona, et al. Rotavirus mortality confirmed by etiologic identification in venezuelan children with diarrea. Pediatr. Infect Dis J. 26 (2007) 393397.

[21] R.B Sack, M Rahman., M. Yunus., E.H. Khan. Antimicrobial resistance in organisms causing diarrheal disease. Clin Infect. Dis. 1 (1997): S102-S105.
[22] G. N. Schroeder and H.Hilbi. Molecular Pathogenesis of Shigella spp.: Controlling Host Cell Signaling, Invasion, and Death by Type III Secretion. Clin.Microbiol.Rev.(2008). 21(1):134-156.

[23] Manual de procedimientos: diagnóstico y caracterización de Shigella spp. Departamento de Bacteriología, Instituto Nacional de Enfermedades Infecciosas A.N.L.I.S. "Dr. Carlos G. Malbrán" WHO global salm surv. (2007).

[24] K. Todar. Shigella and Shigellosis. Department of Bacteriology, University of Wisconsin-Madison. USA (2009). On line: www.textbookofbacteriology.net

E-mail: cesgueba@ec-red.com 